\title{
Valoración de la eficacia de la política de información de la Unión Europea a través de las opiniones y actitudes de la ciudadanía salmantina
}

\author{
Valuation of the effectiveness of the information policy of the European Union \\ through the European Information Centres: the case of Salamanca
}

\section{Yolanda Martín González (1) y Ana B. Ríos Hilario (2)}

(1) Dpto. Biblioteconomía y Documentación (Universidad de Salamanca), c/ Francisco Vitoria 6-16, 37008 Salamanca, ymargon@usal.es (1), anarihi@usal.es (2).

\begin{abstract}
Resumen
Se presenta el informe final de una investigación realizada por el Departamento de Biblioteconomía y Documentación y el Centro de Documentación Europea de la Universidad de Salamanca, con el fin evaluar la eficacia de la actual política de información de la Unión Europea (UE). Previamente se exponen los objetivos generales y específicos que se pretenden alcanzar y se explica la metodología elegida para la consecución de los mismos. Seguidamente se exponen los resultados así como un análisis objetivo de los mismos. Se concluye ofreciendo una serie de recomendaciones consideradas apropiadas con el fin de mejorar la situación informativa actual de la ciudadanía europea.
\end{abstract}

Palabras clave: Política de información. Unión Europea. Salamanca.

\section{Introducción}

En los últimos años, la Unión Europea ha apostado firmemente por el diseño de una política de información y comunicación que sirva, en primer lugar, para aminorar el distanciamiento existente entre sus instituciones y la ciudadanía; en segundo lugar, para impulsar el proyecto de integración europeo el cual se halla sumido, en estos momentos, en una profunda crisis tras el no francés y holandés al Tratado Constitucional.

Varias han sido las campañas de divulgación realizadas y muchos los informes y documentos elaborados por las instituciones europeas con el fin de mejorar sus canales de comunicación con la sociedad. En este sentido basta recordar el programa PRINCE puesto en marcha con motivo de la puesta en circulación de la moneda

\begin{abstract}
The final report of a research made by the Department of Library and Information Science and the European Documentation Center of the University of Salamanca appears, with the aim to evaluate the effectiveness of the present European Union's information policy. Previously, the general and specific objectives are exposed and the methodology that has been chosen for the attainment of such is explained. Next, the results are presented and analysed. As a conclusion, a series of recommendations is offered that are considered appropriate to the purpose of improving the present informative situation of the european citizenship.
\end{abstract}

Keywords: Information policy. European Union. Salamanca.

única o las distintas iniciativas informativas en materia de política medioambiental.

No obstante y pese a los enormes esfuerzos realizados hasta ahora por la Comisión se sigue constatando un claro desconocimiento de los mecanismos puestos en marcha por la Unión con el fin de suministrar información a la opinión pública.

En este contexto se inscribe el presente informe realizado en el marco de una investigación llevado a cabo por un grupo de profesores de la Universidad de Salamanca, miembros en su mayoría del Departamento de Biblioteconomía y Documentación. Asimismo se ha contado con la participación del personal de una de las unidades que conforman la red de información de la Comunidad Europea: el Centro de Documentación Europea de dicha universidad. 


\section{Objetivos}

El principal propósito del estudio era tener una constancia objetiva del grado de información y conocimiento que sobre la Unión Europea posee la opinión pública y de este modo, emitir una valoración contrastada sobre la eficacia de la política de información eurocomunitaria.

El mencionado propósito suponía al mismo tiempo la consecución de los siguientes objetivos específicos:

1. Realizar un análisis de los conocimientos y actitudes de la opinión pública con respecto a la Unión Europea.

2. Contribuir a la información y sensibilización de la población sobre el proceso de integración europeo.

3. Analizar y valorar el grado de conocimiento que la población posee sobre la Unión.

4. Acercar ésta a los ciudadanos concienciándolos de que lo que se decide en Bruselas incide directamente en su vida cotidiana.

5. Orientar al ciudadano sobre los diferentes puntos de información dispuestos por la Unión Europea con el fin de que sepan a cual de ellos dirigirse en función de la temática de su consulta.

6. Hacer más accesible y comprensible a la sociedad los documentos generados por las instituciones europeas.

7. Difundir la importancia del Centro de Documentación Europea como nexo de unión entre la Unión Europea y su ciudadanía.

8. Ofrecer a los ciudadanos información, asesoramiento y respuesta a sus cuestiones sobre la Europa comunitaria.

En definitiva, lo que hemos pretendido ha sido, sobre todo, conocer cual es el nivel de conocimiento que la opinión pública posee con respecto a lo que ocurre en Bruselas y, de este modo, emitir un juicio de valor sobre la eficacia de sus dispositivos en materia informativa. Igualmente, hemos intentado captar el sentimiento general existente con respecto a Europa de un público no experto y lejano a la realidad europea.

\section{Metodología}

La metodología elegida para la consecución de los objetivos anteriormente mencionados fue de tipo cuantitativo y, en concreto, ha sido la encuesta. Se diseñó un cuestionario con preguntas cerradas de dos tipos: de respuesta sugerida y de valoración. También, se incluyó una pregunta de carácter abierto con el fin de que el público pudiera exponer más libremente sus opiniones e inquietudes sobre la utilidad de la información eurocomunitaria.

Las preguntas de tipo cerrado aparecían clasificadas a su vez en tres grupos:

1. Preguntas sobre el perfil del encuestado (sexo, edad, nivel de estudios y situación socioprofesional).

2. Preguntas generales sobre aspectos básicos relativos a la Unión Europea.

3. Preguntas sobre el nivel y acceso a la información eurocomunitaria.

En total, quince fueron los ítems procesados.

Dado que nuestro deseo era obtener una muestra representativa de población lo más amplia posible, optamos por invitar a participar en el estudio a las asociaciones de vecinos de la ciudad de Salamanca. En concreto, se escogieron siete asociaciones en función del área urbana en la que se encontraran localizadas, primando siempre el que estuvieran representadas todas las zonas. La invitación a participar en el estudio se realizó a través del correo postal y también a través de una entrevista personal.

A cada una de las siete asociaciones se les distribuyó el mencionado cuestionario aunque solamente cinco agrupaciones colaboraron con la investigación. Pese a ello obtuvimos una muestra bastante amplia dado que al final conseguimos reunir 605 encuestas cumplimentadas. En todos los casos, fueron las propias asociaciones las encargadas de hacerles llegar a sus socios al cuestionario, el cual fue a su vez repartido en las diferentes actividades que estas agrupaciones organizan en sus locales. Existe la excepción de una asociación que únicamente optó por depositar en un lugar visible de su local los cuestionarios para que la gente interesada los contestara. Este dato aparentemente insignificante ha tenido una importancia notable en el número de encuestas cumplimentadas que después hemos recibido de esta agrupación vecinal. El plazo estipulado para la cumplimentación del cuestionario fue de dos meses desde la fecha en la que se les facilitó. Dado que no se entregó el documento a la vez en todas las asociaciones, el periodo transcurrió entre los meses de noviembre de 2006 y febrero de 2007.

Sin embargo, hemos de señalar que aunque la realización del cuestionario fue la principal metodología utilizada para la realización de la investigación, se realizaron también otras dos actividades de naturaleza diferente pero que fueron un complemento de aquella y a las que 
lógicamente se invitó a participar a las asociaciones de vecinos colaboradoras:

1. La celebración de un curso titulado: "La información en la Unión Europea: política, sistema y redes" celebrado durante el mes de noviembre en la Facultad de Traducción y Documentación. En dicho curso se contó con la participación de hasta diez ponentes distintos de diversas disciplinas que trabajan sobre los ámbitos de la Unión Europea, la información y la ciudadanía. A través de dicho curso, los asistentes pudieron conocer más a fondo la denominada Europa de la información y los distintos canales existentes para informarse y documentarse sobre la Comunidad Europea así como sobre los derechos que su condición de ciudadanos europeos tienen. Todas las conferencias pronunciadas en este evento fueron convenientemente publicadas por la Universidad de Salamanca cubriendo así un lamentable vacío bibliográfico existente sobre este tema.

2. La segunda actividad consistió en la celebración de dos jornadas llevadas a cabo en las sedes de sendas asociaciones de vecinos. La actividad conllevó a su vez la realización de tres tareas distintas: una charla informativa impartida por la Directora de la investigación y por la documentalista del Centro de Documentación Europea de la Universidad salmantina; la organización de un concurso en el que los participantes tenían que adivinar la respuesta de las preguntas que se les planteaba sobre aspectos tales como el color y el número de estrellas que conforman la bandera eurocomunitaria, cual es la institución que defiende los derechos de los ciudadanos europeos, la fecha de creación de las Comunidades Europeas, la fecha de adhesión de España a las mismas, etc.; la distribución de material informativo sobre la Unión Europea proporcionado por el Centro de documentación europea, como son: folletos, pegatinas, cuadernos para colorear, bolígrafos, agendas, etc.

Hemos de señalar que la celebración de estas actividades fue debidamente difundida en los diarios y emisoras de radio locales, lo que nos permitió contar con la participación tanto de niños como de adultos.

\section{Resultados}

Como ya hemos indicado en líneas anteriores, las seiscientas cinco personas que participaron en nuestro estudio tuvieron que responder a un cuestionario conformado por tres grupos de preguntas cerradas con las que además de conocer el perfil del encuestado, obtuvimos información sobre su nivel de conocimiento de la UE y sobre sus actitudes informativas respecto a ésta.

En el último de los grupos de preguntas se incluyó una de carácter abierto. Dada la variedad y relevancia de las respuestas obtenidas, nos ha parecido oportuno dedicar unas líneas aparte a su análisis.

A continuación exponemos los resultados alcanzados en nuestra investigación organizados en función de los tres tipos de preguntas mencionados.

\subsection{Perfil de los encuestados}

El primer conjunto de cuestiones se planteó con el objeto de conocer el perfil de las personas que participaban en el estudio. De este modo, se incluyeron en el cuestionario cuatro preguntas para conocer el sexo, la edad, el tipo de estudios realizados y la actividad profesional de los encuestados.

Los resultados nos indicaron lo siguiente:

1. En primer lugar, respondieron a nuestro cuestionario un mayor número de mujeres $(55,8 \%)$ que de hombres $(44,1 \%)$.

2. En segundo lugar, sobresale el grupo de personas con edades comprendidas entre los 50 y los 64 años $(37,5 \%)$ seguido del grupo que cuenta entre 35 y 49 años (28\%). El resto de encuestados se engloban en los restantes grupos de edad menores y mayores a los mencionados.

3. En tercer lugar, ha sido mayor el número de encuestados que poseían el título de bachiIlerato $(36,7 \%)$ o estudios primarios $(35,9 \%)$. Sin embargo, el porcentaje de personas sin estudios $(7,8 \%)$ o con un primer $(13,2 \%)$ o segundo $(6,5 \%)$ nivel de estudios universitarios ha sido bastante más bajo.

4. En cuarto lugar, la mayor parte de salmantinos que respondieron al cuestionario han sido trabajadores no cualificados $(23,8 \%)$, seguidos de los empleados administrativos y trabajadores de los servicios $(17,0 \%)$ y los técnicos o profesionales asociados a titulaciones no universitarias o no universitarias de carácter postsecundario $(13,1 \%)$.

\subsection{Conocimiento de la Unión Europea}

En el segundo bloque se plantearon cinco cuestiones de carácter general que nos permitieran obtener una idea sobre el conocimiento que los salmantinos, esto es, los ciudadanos de a pie, 
poseen sobre aspectos tan básicos como el nombre del presidente de la Comisión Europea, el valor oficial del euro en pesetas, los diputados con que cuenta España en el Parlamento Europeo, la fecha de incorporación de nuestro país a las Eurocomunidades, así como el cargo que ocupaba Josep Borrell. Es decir, que nos sirvieran para asegurarnos de que la opinión pública conoce aquellos personajes, fechas o aspectos más elementales de la UE y en concreto, referidos a su país.

En todos los casos se dieron cuatro opciones de respuesta posibles incluida la de no sabe / no contesta.

El porcentaje de aciertos en cada una de las preguntas fue el siguiente:

\begin{tabular}{lc}
\hline Pregunta & Porcentaje de aciertos \\
\hline Presidente de la Comisión & 78,8 \\
\hline Valor del $€$ en ptas & 98,5 \\
\hline Eurodiputados & 44,0 \\
\hline $\begin{array}{l}\text { Fecha ingreso de España } \\
\text { en la UE }\end{array}$ & 73,4 \\
\hline $\begin{array}{l}\text { Cargo ocupado por J. } \\
\text { Borrell }\end{array}$ & 63,0 \\
\hline
\end{tabular}

Tabla I. Nivel de conocimiento sobre la UE.

Tal como se desprende de la tabla precedente los salmantinos cuentan con los conocimientos fundamentales -al menos los que nosotros les planteamos y que nos parecen importantessobre la UE y sus actores. En especial, y por lo que nos afecta a nuestra economía, el dato que mejor conocen es la equivalencia de la moneda única en nuestra querida y desde 2002 desaparecida peseta. Solamente el porcentaje de aciertos disminuye en cuanto al número de eurodiputados con los que cuenta España en el hemiciclo comunitario. Ello puede deberse al escepticismo - cuanto no desconocimiento- existente con respecto a la política europea, tal como ha quedado de manifiesto en los últimos comicios celebrados en 2004 y en los que se contó con una tasa baja de participación ciudadana (en torno al $40-42 \%)$.

\subsection{Nivel y acceso a la información de la UE}

De todo el cuestionario, éste era el grupo de preguntas más importante para el desarrollo de nuestra investigación de ahí que contuviera hasta seis cuestiones de tipo cerrado. A ellas se añadió una cuestión de carácter abierto, que por las razones ya aducidas, pasaremos a comentar más tarde.
De las seis preguntas cerradas, las tres primeras se han contemplado en algunas ocasiones en los sondeos de opinión que realiza la oficina del Eurobarómetro asiduamente. Con ellas se pretendía averiguar: si los ciudadanos se consideran informados sobre la UE, qué medios les proporcionan este tipo de información, y que opinión les merece la cobertura dada por los medios de comunicación españoles.

En concreto a la pregunta: "En términos generales, ¿usted se considera informado sobre la UE?", las respuestas ofrecidas fueron las siguientes:

\begin{tabular}{lc}
\hline Pregunta & Respuesta (\%) \\
\hline Muy bien informado & 4,0 \\
\hline Bastante informado & 16,5 \\
\hline Informado & 31,9 \\
\hline Poco informado & 46,4 \\
\hline
\end{tabular}

Tabla II. Consideración sobre la cantidad de información recibida.

La pregunta: "A través de qué medios recibe normalmente información sobre la UE?" ofrecía diferentes opciones de respuesta y los ciudadanos declararon lo que sigue:

\begin{tabular}{lc}
\hline Pregunta & Respuesta (\%) \\
\hline Televisión & 20,5 \\
\hline Internet & 18,8 \\
\hline Radio & 16,2 \\
\hline Prensa escrita & 25,3 \\
\hline $\begin{array}{l}\text { Revistas de } \\
\text { información general }\end{array}$ & 2,5 \\
\hline
\end{tabular}

Tabla III. Medios a través de los que se recibe la información.

A la pregunta de valoración sobre la consideración que les merecía la cobertura dada por los medios de comunicación de masas en España los encuestados contestaron:

\begin{tabular}{lc}
\hline Pregunta & Respuesta (\%) \\
\hline Demasiada & 3,0 \\
\hline Mucha & 8,4 \\
\hline Suficiente & 34 \\
\hline Poca & 38,8 \\
\hline Muy poca & 14,2 \\
\hline
\end{tabular}

Tabla IV. Cobertura dada por los medios de comunicación españoles. 
Las otras tres preguntas planteadas presentan un carácter más novedoso, puesto que no suelen contemplarse en otros estudios similares realizados hasta la fecha. A través de estas cuestiones queríamos saber si los salmantinos conocen y han visitado y/o utilizado los centros e instrumentos informativos dispuestos por la Unión Europea, en concreto, los Centros de Documentación Europea (CDE) y el Portal Europa. Además queríamos saber a dónde se dirigiría un ciudadano en el hipotético caso de que necesitara algún de tipo de información específica sobre Europa.

De este modo, a la pregunta: "¿Conoce y/o ha visitado alguna vez un Centro de Documentación Europea?" los ciudadanos contestaron:

\begin{tabular}{lc}
\hline Pregunta & Respuesta (\%) \\
\hline No lo conozco & 77,5 \\
\hline $\begin{array}{l}\text { Lo conozco pero no lo } \\
\text { he visitado }\end{array}$ & 16,7 \\
\hline $\begin{array}{l}\text { Lo conozco y lo he } \\
\text { visitado }\end{array}$ & 5,3 \\
\hline
\end{tabular}

Tabla V. Conocimiento ciudadano del CDE.

En cuanto a la cuestión: "Si requiriera de algún tipo de información específica sobre la UE, ¿dónde cree usted que podría localizarla en Salamanca?", los datos arrojados fueron los siguientes:

\begin{tabular}{lc}
\hline Pregunta & Respuesta (\%) \\
\hline Ayuntamiento & 9,9 \\
\hline Biblioteca & 9,1 \\
\hline Internet & 34,4 \\
\hline Otros & 6,8 \\
\hline Varios & 6,9 \\
\hline No sabe / & 31,7 \\
no contesta & \\
\hline
\end{tabular}

Tabla VI. Localización de información sobre la UE.

Por último, se les preguntó si conocían y/o habian visitado alguna vez el Portal Europa, a la que respondieron:

\begin{tabular}{lc}
\hline Pregunta & Respuesta (\%) \\
\hline No lo conozco & 75,5 \\
\hline $\begin{array}{l}\text { Lo conozco pero no lo } \\
\text { he visitado }\end{array}$ & 20,5 \\
\hline $\begin{array}{l}\text { Lo conozco y lo he } \\
\text { visitado }\end{array}$ & 3,1 \\
\hline
\end{tabular}

Tabla VII. Conocimiento ciudadano del Portal Europa.

\subsection{Pregunta abierta}

Las preguntas abiertas resultan valiosas en cualquier tipo de estudios puesto que aportan una dimensión diferente al dejar al encuestado que responda libremente mostrando sus inquietudes, satisfacciones o preocupaciones con respecto al tema objeto de la encuesta.

En nuestro caso únicamente 109 personas de las 605 que colaboraron con nuestra investigación respondieron a la pregunta: "¿para qué piensa usted que le podría ser útil obtener información sobre la UE?".

Como es lógico la utilidad de la información eurocomunitaria varía en función de los intereses personales. La edad y la profesión de los encuestados son los aspectos que más han influido a la hora de responder a esta pregunta.

Diversas han sido las formas en que se han expresado los salmantinos, aunque en sus respuestas se observan dos actitudes claramente diferenciadas.

Sobresale la actitud positiva de los salmantinos, que consideran que el estar informado siempre es útil. En el caso de la Unión Europea es especialmente interesante porque nos sirve para saber qué decisiones se toman en Bruselas y nos pueden afectar, para conocer los derechos que poseemos por ser ciudadanos europeos, obtener ayudas y subvenciones, saber en qué consiste y que cambios supone el Espacio Europeo de Educación Superior e incluso para comprobar las diferencias existentes, a nivel económico, social, etc., entre los países miembros de la Unión.

Por otro lado, se constata una actitud negativa entre aquellos ciudadanos que consideran que obtener información sobre Europa no sirve para nada ya que los dirigentes actúan en función de sus propios intereses y únicamente recurren al ciudadano de a pie cuando necesitan su voto. Se observa que el número de respuestas de este tipo aumenta con la edad del encuestado lo que nos hace pensar que ello se deba a un claro escepticismo sobre el denominado sueño europeo.

Un tercer grupo de respuestas las han ofrecido aquellos salmantinos que dicen no saber para qué les podría ser útil esta información, lo que nos resulta hasta cierto punto preocupante.

\section{Conclusiones}

Una vez descritos objetivamente los resultados obtenidos, exponemos las conclusiones a las que un análisis minucioso de los mismos nos ha permitido llegar. Algunas de ellas coinciden 
con las expuestas en los informes del Eurobarómetro; otras, sin embargo, muestran una visión diferente no sólo de aquéllos, sino también de la percepción que nosotros mismos teníamos al iniciar la investigación.

Así, de las respuestas obtenidas, llegamos a las conclusiones siguientes:

1. El papel que juega la UE en el ámbito económico sigue siendo el aspecto del proyecto de integración más conocido por los ciudadanos tal como demuestra el alto nivel de aciertos a la pregunta sobre el valor oficial de la moneda única en pesetas. Ello, como es lógico, se debe a que la política monetaria es una de las políticas eurocomunitarias que más afecta a su vida cotidiana y en particular, a su economía doméstica.

2. En cuanto a la fecha de ingreso de España en las Comunidades Europeas la cantidad de respuestas acertadas creemos se debe a que los grupos de edad más numerosos participantes en este estudio vivieron ese momento histórico, ya que el desconocimiento de este dato iba en aumento según disminuía la edad del encuestado.

3. Curiosamente, el nivel de estudios no afecta al conocimiento que los salmantinos poseen de la UE, lo que indica que la información la obtienen por otros medios que no son los estrictamente académicos. Ello, en nuestra opinión, debería subsanarse, pues la falta de concienciación europeísta se debe, en gran medida, a la poca presencia que la UE tiene en el ámbito académico.

4. No existe una gran diferencia entre el grupo de ciudadanos que se consideran informados y los que se consideran poco informados sobre la UE. En este sentido, nos ha llamado especialmente la atención el que algunos encuestados mostraran una cierta saturación en cuanto a la información que sobre la UE reciben. Este dato resulta hasta cierto punto sorprendente ya que a la UE se le ha achacado, con frecuencia, un déficit informativo que precisamente su política de información y comunicación ha venido a subsanar. En este sentido, podríamos afirmar que dicha iniciativa está consiguiendo dar sus frutos, aunque no hemos de desdeñar el papel jugado por los medios de comunicación en la difusión de información sobre lo que acontece en Bruselas. Los medios multiplicadores de opinión siguen jugando un papel crucial en la transmisión de información, aunque los ciudadanos piensan que no es suficiente; de ahí que estén optando por la utilización de otros medios tecnológicos para informarse sobre la UE o simplemente porque les resulte más cómodo.

5. Las nuevas tecnologías han reducido la importancia estratégica no sólo de los medios de comunicación tradicionales sino de las unidades de información convencionales tales como bibliotecas, centros de documentación e incluso instituciones de la administración pública como los ayuntamientos. La utilización de Internet va ganando puestos frente a la de otros medios como la televisión, la radio o la prensa escrita, responsables hasta hace bien poco de la información eurocomunitaria ante la opinión pública.

6. Al hilo de lo anterior, resulta curioso que un gran porcentaje de ciudadanos desconozcan la que es la fuente de información en Red por antonomasia de la UE, el portal Europa. Ello nos hace pensar que cuando los usuarios buscan información sobre Europa en la Red lo hacen bien a través de la prensa en formato digital bien a través de multibuscadores tipo Google, pero no en los sitios web dispuestos específicamente por la Unión.

7. En Salamanca, existe un desconocimiento más que generalizado del Centro de Documentación Europea. Ello puede deberse a que este tipo de centros se enmarca física y estatutariamente en el ámbito universitario. Sin embargo, la carencia de otro centro de información de la UE en nuestra ciudad, lo convierte en principal referencia informativa y documental sobre el ámbito que nos ocupa. Dado que nuestra pregunta únicamente se refería a esta unidad de documentación concreta, no podemos vaticinar un desconocimiento del conjunto de redes y centros de información eurocomunitarios, aunque mucho nos tememos que así sea.

Todo ello nos llevar a deducir que la política informativa puesta en marcha por la Comunidad está siendo relativamente efectiva, aunque nosotros preferimos ser cautos con esta afirmación, pues no debemos subestimar el papel que juegan los medios de comunicación. No obstante, observamos que los salmantinos sí poseen información general sobre la UE, sus políticas y sus responsables políticos, aunque la busquen en medios distintos a los utilizados hasta ahora. Lo que sí consideramos preocupante es la falta de conocimiento de los centros y dispositivos informativos dispuestos específicamente por la Unión Europea. 


\section{Recomendaciones}

A la vista de los resultados obtenidos, se deducen algunas recomendaciones de acción más que evidentes. Por encima de todas y englobándolas, está la de que, pese a que va mejorando, la política de información sigue siendo deficiente especialmente en lo que se refiere al grado de conocimiento ciudadano sobre sus redes y centros de información y a su presencia en Internet.

Queremos señalar, no obstante, que algunas de las sugerencias que hacemos a continuación ya fueron puestas en práctica por este grupo de investigadores. En nuestra opinión, la situación actual podría verse mejorada si se tuvieran en cuanta algunas recomendaciones tales como:

- Reorientar algunas de las políticas de difusión de las instituciones y organismos europeos. Dichas políticas ponen excesivamente el acento en el papel mediador de los medios de comunicación, cuando en el fondo hay que intentar popularizar las redes y centros de información eurocomunitarias. Estas unidades que responden al principio de descentralización informativa, juegan un rol importantísimo, puesto que -al ubicarse físicamente en las zonas urbanas y rurales, donde los ciudadanos desarrollan su labor cotidiana- hacen que la UE adquiera una imagen más cercana y tangible.

- En el caso concreto del Centro de Documentación Europea de Salamanca, creemos que es la Universidad quien debería poner los medios necesarios para su difusión, habida cuenta de que es su responsable. Sabemos, por nuestra experiencia como responsables de la asignatura: "Documentación Europea" impartida en la Diplomatura de Biblioteconomía y Documentación, que los propios estudiantes universitarios desconocen su existencia, lo que nos hace pensar que con el resto de ciudadanos ocurra lo mismo. Esta situación es del todo lamentable. Sin embargo, sabemos a ciencia cierta que desde el CDE se han puesto en marcha diferentes actividades que sirvieran para darse a conocer no sólo dentro sino también fuera del perímetro universitario. Prueba de ello, es una de las actividades que realizamos en la sede de algunas asociaciones de vecinos salmantinas y que ya hemos comentado en el apartado de metodología.

- Dado que la Red está ganando cada vez más adeptos a la hora de buscar información, aconsejamos se promuevan aquellas actividades destinadas a mostrar y enseñar el manejo de las fuentes de información eurocomu- nitarias. De este modo, los ciudadanos sabrán cuales son los recursos electrónicos más apropiados en función de su demanda informativa. Somos conscientes de que ello implicaría tener un nivel de alfabetización informacional que no se da por igual en todos los grupos de edad. Sin embargo, animamos a los profesionales de la documentación en general y a los que desarrollan su labor en las redes y centros de información eurocomunitarios en particular, a que propongan iniciativas -que pueden ser cursos, seminarios, etc.pues nuestro bagaje profesional nos indica que éstas son escasas tanto en España como en el resto de Europa. Eso sí, debe primar siempre la descentralización divulgativa.

- Pese a que en el Libro Blanco de la Comisión se incide en el papel que juegan las nuevas tecnologías de la información en la difusión del proyecto europeo y a la puesta en práctica de los postulados en él contemplados, con la creación de sitios web dedicados a recoger las inquietudes y opiniones de la ciudadanía, la política informativa sigue sin hacerse visible. Por ello, recomendamos que se haga difusión de la existencia de estos sitios desde dentro pero también desde fuera de la Red para así llegar a un público más numeroso.

- La política de información ha de apoyarse en otras políticas como la educativa. La escasa presencia de la UE en la vida académica de los europeos fomenta, de algún modo, el distanciamiento entre aquélla y la ciudadanía. Los educadores pueden ser unos magníficos difusores del proyecto de integración europeo $y$, por tanto, un apoyo imprescindible para las iniciativas informativas y comunicativas diseñadas en Bruselas. En este sentido, existen ya varias iniciativas de tipo comunicativo dirigidas ex profeso a los colegios de los países miembros. La finalidad de dichas iniciativas es la de conocer qué conocimientos poseen los escolares europeos sobre la Unión y conocer su opinión sobre ella.

- En definitiva, se debe pensar en estrategias de difusión que hagan más accesible la información a los ciudadanos independientemente del perfil socioprofesional que éstos presenten. Sólo de este modo, la Unión Europea conseguirá entusiasmar y comprometer a la ciudadanía con el proyecto europeo al tiempo que ser verdaderamente transparente.

\section{Referencias}

Baigorri Agoiz, Artemio; Fernández Díaz, Ramón; Masa Godoy, Leopoldo (2001). Los universitarios extremeños ante la Unión europea. Análisis de conocimientos y acti- 
tudes. Badajoz: Centro de Documentación e Información Europea de Extremadura, 2001.

Cea D’Ancona, María Angeles. (1996) Metodología cuantitativa: estrategia y técnicas de investigación social. Madrid: Síntesis, D.L. 1996

Comisión Europea. (2006) Eurobarómetro. http://ec.europa. eu/public_opinion/standard_en.htm (2007-05-26).

Comisión Europea (2006). Libro Blanco sobre una política europea de comunicación. // COM. (01.02.2006) 35 final.

Desantes-Guanter, José María. (1996) Teoría y técnica de la investigación científica. Madrid: Síntesis, D.L. 1996.
López Yepes, José. (1995) La aventura de la investigación científica: guía del investigador y del director de la investigación. Madrid: Síntesis, D.L. 1995.

Patronat Català pro Europa (2006). Opina! Contribución de la estructura de acogida Europe Direct del Patronat Català Pro Europa al Libro Blanco de la Comisión Europea sobre una política europea de comunicación. Barcelona: Patronat Català Pro Europa, 2006.

\section{Apéndice}

Con motivo de la investigación que el Departamento de Biblioteconomía y Documentación de la Universidad de



\section{DEPARTAMENTO DE BIBLIOTECONOMÍA Y DOCUMENTACIÓN}

UNIVERSIDAD DE SALAMANCA

Salamanca está llevando a cabo en colaboración con el Centro de Documentación Europea, con el fin de conocer el grado de información de la ciudadanía salmantina con respecto a la Unión Europea, les agradeceríamos colaboraran con nosotros respondiendo al siguiente cuestionario. Para ello señale con una cruz o un círculo la respuesta elegida.

\section{DATOS GENERALES}

1.- Sexo
a) Hombre

b) Mujer

2.- Edad
a) $<20$ años
b) 20 a 34 años
c) de 35 a 49 años
d) de 50 a 64 años
e) $>65$ años

3.- Estudios
a) Sin estudios
b) Primarios completos
c) Bachillerato elemental o asimilados
d) Diplomado universitario o asimilado (estudios profesionales de grado medio, peritajes, magiste- rio, enfermería, etc.).
e) Licenciado, Ingeniero, Arquitecto, E. Su- perior Militar o asimilado.

4.- Profesión

a) Directores o gerentes o empresas para la Administración Pública

b) Técnicos o profesionales asociados a titulaciones universitarias o no universitarias de carácter postsecundario

c) Empleados administrativos y trabajadores de los servicios

d) Trabajadores cualificados en agricultura y pesca

e) Trabajadores cualificados y operadores de máquinas en la industria, la construcción y la minería

f) Trabajadores no cualificados

g) Profesionales de las Fuerzas Armadas h) Personas que no han tenido un trabajo remunerado

\section{CONOCIMIENTO DE LA UE}

1.- El Presidente de la Comisión Europea es:
a) Durao Barroso
b) Javier Solana
c) F. Mayor Zaragoza
d) No sabe / No contesta

2.- El valor oficial aproximado del euro en ptas es:
a) 166,39 ptas.
b) 100,39 ptas.
c) 150,39 ptas
d) No sabe / No contesta

3.- El número de eurodiputados con los que cuenta España en el Parlamento Europeo es:
a) 60
b) 54
c) 30
d) No sabe / No contesta

4.- ¿En qué año España se incorporó a la UE?
a) 1975
b) 1982
c) 1986
d) No sabe / No contesta

5.- ¿Qué ha ocupado Josep Borrell?
a) Comisario europeo por España
b) Presidente del Parlamento Europeo
c) Secretario General del Consejo de la UE
d) No sabe / No contesta través de las opiniones y actitudes de la ciudadanía salmantina. // Ibersid. (2007) 43-51. ISSN 1888-0967. 
NIVEL Y ACCESO A LA INFORMACIÓN EUROCOMUNITARIA

1.- En términos generales, ¿usted se considera informado sobre la UE?
a) Muy bien informado
b) Bastante informado
c) Informado
d) Poco informado

2.- ¿A través de qué medios recibe normalmente información sobre la UE?
a) televisión
b) Internet
c) radio
d) periódicos
e) revistas de información general

3.- Considera que la cobertura dada por los medios de comunicación de masas en España es:
a) Demasiada
b) Mucha
c) Suficiente
d) Poca
e) Muy poca

4.- ¿Conoce y/o ha visitado alguna vez un Centro de Documentación Europea?
a) No lo conozco
b) Lo conozco pero no lo he visitado
c) Lo conozco y lo he visitado alguna vez

5.- Si requiriera de algún tipo de información específica sobre la UE, ¿dónde cree usted que podría localizarla en Salamanca?
a) en el Ayuntamiento
b) en una biblioteca
c) en Internet
d) Otros
e) No sabe / No contesta

6.- ¿Conoce y/o ha utilizado alguna vez el Portal Europa?

a) No lo conozco

b) Lo conozco pero no lo he utilizado

c) Lo conozco y lo he utilizado

7.- ¿Para qué piensa usted que le podría ser útil obtener información sobre la UE?

Gracias por colaborar con nosotros y posibilitar que la información sobre la Unión Europea llegue cada día a más personas. 